\title{
A tecnologia digital nas Diretrizes Curriculares dos Cursos de Design: um estudo da abordagem tecnológica na formação do futuro profissional
}

\section{Digital technology in the curricular guidelines of Design courses: a study of the technological approach in the formation of the future professional}

\author{
Flavia Lumi Matuzawa \\ Doutoranda em Design pela Universidade Federal de Santa Catarina (UFSC). Professora na Universidade do Sul de \\ Santa Catarina (UNISUL) - fla.matuzawa@gmail.com - orcid.org/0000-0001-5481-3435 \\ Ana Leticia Oliveira do Amaral \\ Doutoranda em Design pela Universidade Federal de Santa Catarina (UFSC) - amaral.analeticia@gmail.com - \\ orcid.org/0000-0002-5270-9105 \\ Berenice Santos Gonçalves
}

Doutora em Engenharia de Produção pela Universidade Federal de Santa Catarina (UFSC). Professora na Universidade Federal de Santa Catarina (UFSC) - berenice@cce.ufsc.br - orcid.org/0000-0002-0740-4281

\section{Roseli Zen Cerny}

Doutora em Educação-Currículo pela Pontifícia Universidade Católica de São Paulo (PUC/SP). Professora na Universidade Federal de Santa Catarina (UFSC) - rosezencerny@gmail.com - orcid.org/0000-0001-7882-8551

\section{Marina Bazzo de Espíndola}

Doutora em Educação pela Universidade Federal de Santa Catarina (UFSC). Professora na Universidade Federal de Santa Catarina (UFSC) - marinabazzo@gmail.com - orcid.org/0000-0003-3039-5528

\section{Resumo}

A partir da Resolução CNE/CES no 5, de 8 de março de 2004, que aprova as Diretrizes Curriculares Nacionais para cursos de Graduação em Design, faz-se neste artigo uma análise do documento sob a ótica de como o mesmo compreende o conceito de tecnologia, currículo e formação crítica no uso da tecnologia digital na expectativa de visualizar um futuro designer preparado para atuar no contexto contemporâneo. Os desafios requerem um profissional crítico capaz de perceber os problemas e desenhar soluções, envolvendo as qualidades da tecnologia digital para potencializar suas soluções projetuais. $O$ estudo conclui que a tecnologia não tem uma ênfase explícita em sua abordagem na DCN, o que pode refletir diretamente no perfil de um profissional crítico ao uso das tecnologias digitais.

Palavras-chave: Design. Tecnologia Digital. Formação crítica. Diretrizes Curriculares Nacionais.

\begin{abstract}
Based on CNE/CES Resolution No. 5 of March 8, 2004, which approves the National Curricular Guidelines for Graduation Courses in Design, this article analyzes the document from the point of view of how it understands the concept of technology, curriculum and critical training in the use of technology in the expectation of visualizing a future designer prepared to act in the current context. Challenges require a critical professional who can understand the problems and design solutions involving the potential of technology to expand the potential and reach of solutions. The study concludes that the technology does not have an emphasis in its approach in the DCN which can reflect directly in the profile of the one of critical professional in the use of the technology.
\end{abstract}

Keywords: Design. Digital Technology. Critical formation. National Curricular Guidelines.

Recebido em: 21/05/2019

Aceito em: 26/09/2019 
A tecnologia digital nas Diretrizes Curriculares dos Cursos de Design: um estudo da abordagem tecnológica na formação do futuro profissional

Flavia Lumi Matuzawa, Ana Leticia Oliveira do Amaral, Berenice Santos Gonçalves, Roseli Zen Cerny, Marina Bazzo de Espíndola

\section{INTRODUÇÃO}

O ensino superior de Design, assim como em muitas outras áreas, se depara com diversos desafios potencializados pela mobilidade e interatividade das tecnologias digitais, como as mudanças nas relações sociais, tecnológicas, econômicas e industriais. Consequentemente, faz-se necessária a formação de um profissional capaz de utilizar conscientemente os recursos tecnológicos ao identificar, selecionar, analisar e aplicar as soluções mais adequadas aos projetos desenvolvidos.

Este cenário de constantes mudanças, e em especial no contexto dos cursos de Design, impactam diretamente na concepção dos currículos. Contudo, por mais que um "esforço heróico no sentido de atualizar currículos, introduzir novas disciplinas e promover discussões sobre questões da atualidade vem sendo empreendido ao longo dos anos por muitos docentes e pesquisadores" (COUTO, 2008, p. 69), se faz necessário repensar os processos de formação da área de Design frente a expressiva presença da tecnologia digital.

Perceber o perfil do profissional contemporâneo nesta área de atuação envolve, dentre as diferentes variáveis, considerar as tendências e demandas do mercado nacional e global e compreender como o meio acadêmico têm preparado este profissional com conhecimento crítico e técnico, habilidades e competências específicas à área.

Este trabalho se contextualiza na compreensão de como a tecnologia digital é abordada no documento oficial que apresenta orientações quanto a conteúdos, habilidades e competências para os cursos na área do Design. Ou seja, como as Diretrizes Curriculares Nacionais (DCN) para os cursos de Design compreendem a formação do designer e como ela aponta para uma formação contextualizada ao mundo tecnológico e cada vez mais digital.

Diante desse contexto, para as discussões propostas a esta análise bibliográfica e documental, será utilizada a Resolução CNE/CES no 5, de 8 de março de 2004, a qual aprova as Diretrizes Curriculares Nacionais para Cursos de Graduação em Design (BRASIL, 2004), por ser a última resolução publicada até o momento da construção desta pesquisa. Com base neste documento, o artigo trará um estudo crítico sobre a formação do designer contemporâneo ao realizar uma reflexão acerca da compreensão em torno do que é tecnologia digital para este curso e como ela se insere como conteúdo para o processo de formação profissional do designer. A partir de uma análise do conteúdo da referida DCN, foram definidas categorias de análise: como 
A tecnologia digital nas Diretrizes Curriculares dos Cursos de Design: um estudo da abordagem tecnológica na formação do futuro profissional

Flavia Lumi Matuzawa, Ana Leticia Oliveira do Amaral, Berenice Santos Gonçalves, Roseli Zen Cerny, Marina Bazzo de Espíndola

formação crítica, conceito de ferramenta e conceito de currículo para servir como referenciais de estudo (BARDIN, 2016).

\section{O CURRÍCULO E SUA RELAÇÃO COM AS DIRETRIZES CURRICULARES}

Falar em Diretrizes Curriculares para cursos de graduação requer um olhar anterior para o que se expressa na Lei de Diretrizes e Bases da Educação Nacional (LDB) em seu Capítulo IV, que trata do Ensino Superior. Este é um dos documentos que apresentam orientações para a formação na educação superior e que:

fundamentam o processo de formação na educação superior através do desenvolvimento de competências e habilidades; do aperfeiçoamento cultural, técnico e científico do cidadão; da flexibilização dos currículos; da implementação de Projetos Pedagógicos inovadores, numa perspectiva de mudança para a formação profissional (FERNANDES; XAVIER; CERIBELLI; BIANCO; MAEDA; RODRIGUES, 2005, p. 443).

A partir da LDB desdobra-se o desenvolvimento de Diretrizes Curriculares específicas para diversos cursos de ensino superior no Brasil, de modo que se destaca neste trabalho, a Diretriz Curricular Nacional (DCN) para Cursos de Design, de 2004, por meio da resolução no 3 de 8 de março do referido ano. O documento passa a nortear os currículos dos cursos de Design no país, trazendo referenciais para o perfil do futuro profissional, bem como os conteúdos relevantes para sua formação.

Sacristán (2013, p. 16) fundamenta que "o currículo tem o sentido de construir a carreira do estudante e, de maneira mais concreta, os conteúdos deste percurso, sobretudo sua organização, aquilo que o aluno deverá aprender e superar e em que ordem deverá fazê-lo". O autor afirma que o conceito inicial de currículo relaciona-se a um entendimento de selecionar conteúdos e organizá-los em uma ordem de classificação de conhecimentos. Estes conteúdos ficam organizados a ação de ensinar cubrir o que se acredita que deva ser ensinado.

Ao abordar a organização do currículo e a forma de estabelecer conteúdos, níveis de ensino, território das disciplinas etc., Sacristán (2013, p. 20) aponta para o entendimento de que o currículo torna-se um recipiente não neutro de conteúdos, pois ele "se comporta como um instrumento que tem a capacidade de estruturar a escolarização, a vida nos centros educacionais e as práticas pedagógicas, pois dispõe, transmite e impõe regras, normas, numa ordem que são determinantes". De acordo com o autor, a não neutralidade de conteúdos orienta, portanto, a formação do profissional em questão. Apesar da atualização de diretrizes curriculares compreender 
A tecnologia digital nas Diretrizes Curriculares dos Cursos de Design: um estudo da abordagem tecnológica na formação do futuro profissional

Flavia Lumi Matuzawa, Ana Leticia Oliveira do Amaral, Berenice Santos Gonçalves, Roseli Zen Cerny, Marina Bazzo de Espíndola

um trabalho complexo, o qual requer tempo para discutir e sedimentar pontos de ajustes importantes, uma reflexão sobre quais contextos, regras e normas estão pautados, as atuais diretrizes curriculares pode ser oportuna, considerando que hoje, a sociedade de modo geral está imersa em um desenvolvimento tecnológico.

O contexto do Design conta com tecnologias digitais que possibilitam soluções inovadoras aos projetos que talvez, em 2004 - no contexto da homologação do documento - não eram tão expressivos. Uma vez que o currículo segue as DCN, cabe a reflexão sobre quais conteúdos e qual o perfil as diretrizes almejam para os futuros profissionais.

\section{O ENSINO DO DESIGN BRASILEIRO NO CENÁRIO ATUAL}

Julia (2001) defende que a cultura escolar não pode ser estudada sem a análise das relações conflituosas ou pacíficas mantidas por ela, com o conjunto das culturas que lhe são contemporâneas. Nesse sentido, cabe abordar uma breve reflexão sobre o início do ensino do Design no Brasil, buscando auxiliar na compreensão de aspectos presentes no cenário atual.

Segundo Freitas (2010), a movimentação em torno da criação do Museu de Arte de São Paulo (MASP), em 1950, serviu para o diagnóstico da lacuna existente entre o desenvolvimento da indústria brasileira e a ausência do Design no Brasil. Com isso, o Instituto de Arte Contemporânea (IAC) foi concebido e instalado no MASP sendo uma iniciativa repercutida no cenário empresarial, industrial e político brasileiro, visto que construiu uma corrente de pensamento com a qual os intelectuais e políticos brasileiros trabalhariam posteriormente: a institucionalização efetiva do ensino superior de Design no Brasil (NIEMEYER, 2000; FREITAS, 2010).

Além do IAC, houve outras tentativas de implantação do ensino superior de Design entre 1950 e 1960 no Museu de Arte Moderna (MAM) e no Instituto de Belas Artes, as duas no Rio de Janeiro. Essas tentativas seguiram o padrão europeu com o intuito de desenvolver a indústria brasileira. Para Moraes (2014), o Design europeu calcado no funcionalismo e racionalismo parecia trazer soluções diante das necessidades de uma industrialização ainda iniciante no país.

Nesse contexto, em 1962 no Rio de Janeiro, é criada a Escola Superior de Desenho Industrial (ESDI), que assim como outras escolas de Design, teve como principais influências a escola Bauhaus e a Escola de ULM. Para Hatadani, Andrade e Silva (2010), a criação da ESDI pode ser vista como decorrência de uma série de fatores políticos, econômicos e sociais, estando diretamente ligada à ideologia nacional-desenvolvimentista dos anos 1950, uma época de crenças 
Flavia Lumi Matuzawa, Ana Leticia Oliveira do Amaral, Berenice Santos Gonçalves, Roseli Zen Cerny, Marina Bazzo de Espíndola

modernistas. A ESDI por ter sido considerada uma proposta de ponta, se tornou referência para a criação de diversas faculdades de Design no Brasil e no desenvolvimento de seus currículos (ANGÉLICO; OLIVEIRA, 2017).

Entretanto, Moraes (2008) afirma que o modelo racionalista de ensino importado das escolas europeias, não condiz com o cenário contemporâneo brasileiro, uma vez que os designers na atualidade encontram novos desafios de atuação devido ao estabelecimento de um novo quadro mundial, que passa de um cenário estático para um cenário dinâmico. Além disso, Silva (2017) defende que o cenário brasileiro do Design apresenta o designer generalista resultante do generalismo das diretrizes norteadoras.

\section{AS DCN PARA A GRADUAÇÃO EM DESIGN E SUA RELAÇÃO COM A TECNOLOGIA DIGITAL}

As DCN voltadas às graduações em Design apresentam orientações gerais para o desenvolvimento do curso por meio do Projeto Pedagógico (PP), o qual deve explicitar informações acerca do perfil do formando, habilidades e competências, orientações sobre estágio supervisionado, atividades complementares, sistema de avaliação, bem como informações acerca da oferta da matriz curricular proposta pela Instituição. O referido documento foi homologado em 2004 e está em vigor até o presente momento, portanto, este é o documento considerado na análise deste artigo.

Segundo Jankevicius e Humerez (2015, p. 5):

A orientação estabelecida pela Lei de Diretrizes e Bases da Educação, no que tange ao ensino em geral e ao ensino superior em especial, aponta no sentido de assegurar maior flexibilidade na organização de cursos e carreiras, atendendo à crescente heterogeneidade tanto da formação prévia como das expectativas e dos interesses dos alunos.

Essa flexibilidade na organização de cursos e carreiras está prevista em Brasil (2004, p. 2) para o Curso de Graduação em Design: “os Projetos Pedagógicos do curso de graduação em Design poderão admitir modalidades e linhas de formação específica, para melhor atender às necessidades do perfil profissiográfico que o mercado ou a região assim exigirem".

Neste sentido, compreende-se a possibilidade de localizar PPs com abordagens diferenciadas, dependendo da região e cultura na qual os mesmos atendem. Essas especificidades podem ser expressas em termos da formação específica do designer, seja para o Design Gráfico, Design de Produto ou Industrial, por exemplo. Independente do perfil se parte do princípio da 
Flavia Lumi Matuzawa, Ana Leticia Oliveira do Amaral, Berenice Santos Gonçalves, Roseli Zen Cerny, Marina Bazzo de Espíndola

existência de eixos comuns em termos de conteúdos básicos, específicos e teórico-práticos (BRASIL, 2004). Embora a análise de PP não seja o foco do presente artigo, a possibilidade de organizar o PP e olhar (não somente para o perfil profissional como também para as necessidades de mercado e região), permite, por exemplo, perceber que a tecnologia está incorporada em diversas áreas de formação e na sociedade em geral. Assim, é inevitável considerar esta realidade na formação do futuro designer.

Quanto à compreensão e abordagem ao uso da tecnologia, faz-se necessária a clareza a respeito de como a DCN percebe a inserção da tecnologia digital na formação de designers. Andrew Feenberg é um filósofo cujo trabalho tem destaque quanto à sua reflexão filosófica sobre a tecnologia. Feenberg (apud LOPES, 2015) elabora um espectro das concepções sobre a tecnologia com elementos de interesse para a análise deste artigo.

No quadro 1, a primeira linha horizontal estabelece a relação homem-tecnologia e a primeira coluna indica a relação valor-tecnologia. As células internas da tabela (determinismo, instrumentalismo, substantivismo e teoria crítica) mostram os possíveis cruzamentos entre a relação homem-tecnologia e qual a relação valor-tecnologia e identificam distintas posições na filosofia da tecnologia pensadas a partir dos parâmetros de relação homem-tecnologia e valortecnologia.

\begin{tabular}{|c|c|c|}
\hline \multicolumn{2}{|c|}{ Quadro 1 - Espectro das concepções sobre a tecnologia. } \\
\hline $\begin{array}{c}\text { A tecnologia é } \\
\text { Neutra } \\
\text { (separação completa entre } \\
\text { meios e fins) }\end{array}$ & Autônoma & Humanamente controlada \\
\hline $\begin{array}{c}\text { Não neutra axiologicamente } \\
\text { (carregada de valores) }\end{array}$ & Determinismo & Teoria crítica \\
\hline
\end{tabular}

Fonte: adaptado de Feenberg (2010).

Feenberg (2010) afirma que o determinismo pode ser compreendido na medida em que a tecnologia não é controlada pelos humanos, mas, do contrário, controla e molda a sociedade às exigências do contexto que os cerca. $O$ instrumentalismo vê a tecnologia como uma ferramenta, ou instrumento controlada pelas pessoas atender suas necessidades. A teoria substantiva não faz suposições sobre as necessidades que a tecnologia atende e não é otimista, ela é crítica. Neste 
A tecnologia digital nas Diretrizes Curriculares dos Cursos de Design: um estudo da abordagem tecnológica na formação do futuro profissional

Flavia Lumi Matuzawa, Ana Leticia Oliveira do Amaral, Berenice Santos Gonçalves, Roseli Zen Cerny, Marina Bazzo de Espíndola

quadrante, a tecnologia é vista como ameaçadora, uma distopia, onde, em uma visão extrema, a poderia converter a humanidade em meros dentes de engrenagens de uma máquina. Por fim, a teoria crítica, e à qual as autoras se posicionam a favor, que apesar de reconhecer as consequências catastróficas que o desenvolvimento tecnológico pode tomar se reforçada pela substantivismo, consegue pensar uma realidade melhor para se viver. Nesta concepção, o problema não está na tecnologia como tal, senão no fracasso das pessoas até agora "em inventar instituições apropriadas para exercer o controle humano da tecnologia. Poderíamos adequar a tecnologia, todavia, submetendo-a a um processo mais democrático no design e no desenvolvimento" (FEENBERG, 2010, p. 9).

Assim como o determinismo é transformado pela ideia da não neutralidade axiológica (onde os meios formam um modo de vida que inclui fins), a teoria crítica da tecnologia também o faz com o instrumentalismo (LOPES, 2015). É a partir deste contexto, entre determinismo e teoria crítica, que o Feenberg trabalha um conceito chave de sua filosofia da tecnologia, a chamada teoria da ambivalência da tecnologia, na qual o que está em jogo não é "apenas o alcance limitado dos usos permitidos por qualquer design técnico, mas o número completo de efeitos dos sistemas tecnológicos como um todo" (FEENBERG, 1999 apud LOPES, 2015). Seguindo este conceito, Feenberg entende a tecnologia não como algo em si mesmo, mas como um processo com suas instâncias, - ao que Lopes (2015, p.125) afirma que "a tecnologia aparece aí, portanto, como um campo de batalha, e não como um destino irrevogável".

Uma vez que, na teoria crítica a tecnologia é compreendida como um processo e não como um mero instrumento, acredita-se encontrar neste quadrante a visão desejada que converge para o entendimento do futuro designer frente à apropriação da tecnologia digital em seu contexto profissional. Utilizar a tecnologia digital meramente como um instrumento, pode tender ao reducionismo tecnicista que pouco promove uma atividade instigadora e criativa - que reflete a natureza do Design.

A postura criativa e do designer reforça a importância de seu papel frente à sociedade marcada por uma forte presença de tecnologias digitais, as quais se consolidam na forma de se comunicar de maneira interdisciplinar, projetar soluções e desenvolver na prática estas soluções. 


\section{COMPREENDENDO O QUE É CURRÍCULO}

Silveira, Bertoni e Ribeiro (2016) bem exploram o contexto de base que se reflete na atual DCN do curso de Design. Os autores trazem um histórico onde o ensino superior do Design está preso a conceitos tradicionais que remontam da sociedade industrial, a qual priorizava "majoritariamente, o ensino de habilidades técnicas que limitam o potencial reflexivo e crítico dos alunos" (SILVEIRA; BERTONI; RIBEIRO, 2016, p. 22).

Muito além de um currículo que aborda a tecnologia digital de forma esporádica, a DCN precisa perceber o contexto mercadológico atual onde existem startups, micros e pequenas empresas, cujas estratégias de desenvolvimento devem ser criativas e empreendedoras. Ao encontro dessa realidade, Silveira, Bertoni e Ribeiro (2016) afirmam que, com a difusão das tecnologias digitais de informação e comunicação (TDIC), há demandas por novas abordagens dos docentes que, ao invés de serem vistos como os detentores exclusivo do conhecimento assumem uma posição que promove "uma relação mais viva, dinâmica e colaborativa nas aulas, até mesmo a partir de proposições e indagações dos próprios estudantes, quando engajados" (SILVEIRA; BERTONI; RIBEIRO, 2016, p. 23).

O currículo precisa refletir a visão de futuros profissionais que farão parte de equipes multidisciplinares na sua atuação profissional e para isso é necessário saberem construir um discurso verbal e científico coerente e inteligente, tanto quanto ter criatividade, capacidade projetual e de expressão visual (BONSIEPE, 2011; WHITELEY, 1998 apud SILVEIRA; BERTONI; RIBEIRO, 2016). Ainda refletidos no currículo, os estudantes precisam ser envolvidos no desenvolvimento de competências que vão além de reprodução de objetos e que promovam o uso de métodos científicos, pensamento analítico, lógico e retórico, e habilidades de resolução de problemas (SILVEIRA; BERTONI; RIBEIRO, 2016).

Alcançar essas práticas, requer ajustes no currículo. Couto (2008, p. 29) confirma este desafio ao dizer: "currículos são programas de longo prazo e modificações em sua estrutura devem ser orientadas não apenas pela experiência cristalizada no passado, mas também por prognósticos ou possíveis cenários futuros".

A tecnologia digital precisa ser compreendida com ênfase nas diretrizes de modo que aponte possibilidades de ser utilizada como um processo, e não uma ferramenta técnica pontual, que possa contribuir ao desenvolvimento de soluções criativas e inovadoras para os projetos em 
A tecnologia digital nas Diretrizes Curriculares dos Cursos de Design: um estudo da abordagem tecnológica na formação do futuro profissional

Flavia Lumi Matuzawa, Ana Leticia Oliveira do Amaral, Berenice Santos Gonçalves, Roseli Zen Cerny, Marina Bazzo de Espíndola

Design. Dessa maneira, rever qual a compreensão de "tecnologia" é assumida nas DCN, é crucial para orientar os conteúdos tanto básicos quanto específicos, bem como as práticas projetuais.

\section{COMPREENDENDO A FORMAÇÃO CRÍTICA}

A compreensão no emprego da tecnologia digital na formação do futuro designer está intrinsecamente relacionada com o ensino dos conteúdos no curso de Design. Conforme o Quadro 1, perceber a tecnologia na perspectiva do determinismo e instrumentalismo dificilmente levará ao perfil de um profissional crítico com uma visão aguçada acerca do uso da tecnologia digital no desenvolvimento de suas soluções projetuais. Aqui, cabe ressaltar que o campo do Design ainda se encontra preso ao paradigma da sociedade industrial, muitas vezes priorizando o ensino de habilidades técnica que podem restringir o potencial crítico, reflexivo e criativo dos alunos (MORAES, 2014).

Para sair desta perspectiva e partir para uma formação que priorize um profissional capacitado para atuar em um contexto marcado pela presença da tecnologia digital, Silveira, Bertoni e Ribeiro (2016) acreditam que é sensato propor alternativas futuras diferentes do que se propõe hoje nos PP.

Convém atentar, porém, que ajustes que venham a ser realizados devem basear-se na pesquisa e na teoria do design, em referências pedagógicas atuais e na filosofia geral do design, valorizando não só a técnica e os aspectos científicos do campo, mas, sobretudo, a ética e a estética (SILVEIRA; BERTONI; RIBEIRO, 2016, p. 24).

Após apresentar um estudo consistente acerca do ensino do Design, Silveira, Bertoni e Ribeiro (2016) apresentam algumas premissas para o ensino do Design, das quais destaca-se fomentar o pensamento crítico reflexivo. Esta premissa parece vir ao encontro da formação crítica necessária aos estudantes de Design visando o uso apropriado da tecnologia digital. Para os autores, o pensamento crítico é compreendido como:

a capacidade dos sujeitos de identificar, analisar, avaliar e raciocinar sobre argumentos a fim de se chegar a uma conclusão lógica e racional de forma clara, exata, precisa, relevante, profunda, abrangente e lógica, em resposta a uma observação, experiência, argumento ou expressão verbal ou escrita (SILVEIRA; BERTONI; RIBEIRO, 2016, p. 26).

Essa habilidade é relevante não somente ao processo de resolução de problemas projetuais, como na aplicação correta da tecnologia digital nos projetos. A partir da compreensão 
A tecnologia digital nas Diretrizes Curriculares dos Cursos de Design: um estudo da abordagem tecnológica na formação do futuro profissional

Flavia Lumi Matuzawa, Ana Leticia Oliveira do Amaral, Berenice Santos Gonçalves, Roseli Zen Cerny, Marina Bazzo de Espíndola

de currículo, tecnologia e formação crítica, parte-se para o estudo proposto neste artigo, perceber essas três categorias de análise nas Diretrizes Curriculares Nacionais do Curso de Design.

\section{ANÁLISE}

Na leitura das DCN para o curso de Design, percebe-se uma formação conectada às diferentes manifestações práticas e tecnológicas aplicadas às áreas da graduação, porém mais diretamente explícitas para cursos no nível de pós-graduação lato sensu, conforme consta no Art. 2으, § 1ํ, VIII. Para o nível de graduação, as DCN associam ao futuro profissional uma visão setorial relacionada ao mercado, materiais, processos produtivos e tecnologias em geral. Se considerar a tecnologia especificamente, parece estar envolta diferentes possibilidades de atuação do designer desde o mobiliário, joalheria, identidade visual até softwares e outras manifestações regionais. Ao se referir ao uso de software supõe-se que a tecnologia digital aparece com uma abordagem específica, no uso de um produto específico que poderá auxiliar em atividades isoladas e não como um processo rico em possibilidades.

Quanto ao currículo, há menção sobre como percebem a organização dos conteúdos básicos, específicos e teórico-práticos em linhas gerais, não aparece especificamente o entendimento da tecnologia digital como um processo ou mesmo como um instrumento. Há sim, a compreensão de que este conceito possa estar presente dentro de assuntos específicos dos conteúdos. Nas poucas menções sobre tecnologia encontradas na DCN, uma delas diz respeito ao perfil do formando, onde afirma:

Art. 3o O curso de graduação em Design deve ensejar, como perfil desejado do formando, capacitação para a apropriação do pensamento reflexivo e da sensibilidade artística, para que o designer seja apto a produzir projetos que envolvam sistemas de informações visuais, artísticas, estéticas culturais e tecnólogicas, observados o ajustamento histórico, os traços culturais e de desenvolvimento das comunidades bem como as características dos usuários e de seu contexto sócio-econômico e cultural (BRASIL, 2004, p. 25, grifo nosso).

Ademais as tecnologias são novamente mencionadas ao tratar das atividades complementares no Art. $8^{\circ}$, o qual preconiza que essas atividades possibilitem o reconhecimento do conhecimento, competências e habilidades do aluno especialmente nas relações com o campo de trabalho e com as diferentes manifestações e expressões culturais e artísticas, com as inovações tecnológicas e ações de extensão junto à comunidade. 
Flavia Lumi Matuzawa, Ana Leticia Oliveira do Amaral, Berenice Santos Gonçalves, Roseli Zen Cerny, Marina Bazzo de Espíndola

Contudo, não se percebe uma clareza nas orientações do documento quanto ao emprego do potencial da tecnologia de modo geral, nem de tecnologias digitais no que se refere às habilidades e competências necessárias. Acredita-se que essa perspectiva pode estar contemplada, quando o documento cita, no Artigo 4, item I a "capacidade criativa para propor soluções inovadoras, utilizando domínio de técnicas e processos de criação", compreendendo que em domínio de técnicas e processos de criação esteja contemplado o uso de algum recurso tecnológico.

De modo geral, percebe-se que o documento prevê diversas habilidades e competências essenciais para o desenvolvimento de projetos e relações interpessoais, entretanto, não há ênfase na tecnologia digital em si. Contudo, entende-se que tanto em processos de criação, de desenvolvimento quanto em processos de produção ligados ao Design contemporâneo é possível encontrar a presença da tecnologia digital.

Com relação à formação crítica, as DCN contemplam em seu artigo 3ํㅜ, como pode ser visto na citação acima, um cuidado para a capacitação do futuro profissional no que se refere ao pensamento reflexivo e da sensibilidade artística. Associada a esta competência, o documento espera que o profissional esteja apto para desenvolver as devidas soluções sem perder de vista "o ajustamento histórico, os traços culturais e de desenvolvimento das comunidades, bem como as características dos usuários e de seu contexto sócio-econômico e cultural” (BRASIL, 2004, p. 25).

\section{CONSIDERAÇÕES FINAIS}

Para orientar o estudo da DCN para o curso de Design, realizou-se a análise documental do referido documento a partir das seguintes categorias: (a) conceito de tecnologia; (b) conceito de currículo e (C) formação crítica quanto ao uso da tecnologia digital que a DCN preconiza.

Percebeu-se que a DCN aborda com maior clareza a formação crítica do estudante, mas não necessariamente quanto ao uso da tecnologia digital, assim como o conceito de tecnologia não aparece devidamente explícito para atender as demandas atuais da sociedade. Pode-se presumir que a tecnologia digital está embutida em termos de habilidades e competências, uma vez que não é explícito a menção à apropriação tecnológica. Assim como afirmam Moreira, Sales, Barbosa e Aguilar (2016), há um descompasso entre teoria, ensino e a prática do Design no Brasil, isso pode ser resultante de um discurso ainda mutável sobre o conceito, abrangência, e os limites do Design, bem como a dimensão das habilidades e competências do profissional. 
A tecnologia digital nas Diretrizes Curriculares dos Cursos de Design: um estudo da abordagem tecnológica na formação do futuro profissional

Flavia Lumi Matuzawa, Ana Leticia Oliveira do Amaral, Berenice Santos Gonçalves, Roseli Zen Cerny, Marina Bazzo de Espíndola

Como análise futura, as autoras entendem ser necessário debruçar-se sobre os projetos pedagógicos para avaliar a presença da tecnologia digital e como ela é promovida no currículo dos cursos de Design, ou seja, se interpretada do ponto de vista instrumentalista ou como um processo crítico na formação do designer.

\section{REFERÊNCIAS}

ANGÉLICO, Ana Maria Duarte; OLIVEIRA, Mirtes Cristina Marins de. O ensino do design no Brasil: currículos, ideologias e contemporaneidade. Educação, Cultura e Sociedade, Sinop, v. 2, n. 7, p. 604-615, dez. 2017. Disponível em:

http://sinop.unemat.br/projetos/revista/index.php/educacao/article/view/2629. Acesso em: 04 nov. 2019.

BARDIN, Laurence. Análise do conteúdo. São Paulo: Edições 70, 2016.

BRASIL. Ministério da Educação. Conselho Nacional de Educação Câmara de Educação Superior. Resolução no 5, de 8 de março de 2004. Aprova as Diretrizes Curriculares Nacionais do Curso de Graduação em Design e dá outras providências. Diário Oficial da União: seção 1, Brasília, DF, ano 142, n. 50, p. 24-25, 15 mar. 2019.

COUTO, Rita Maria de Souza. Escritos sobre ensino de Design no Brasil. Rio de Janeiro: RioBook's, 2008.

FEENBERG, Andrew. O que é a filosofia da tecnologia? In: NEDER, Ricardo T. (org.). Andrew Feenberg: racionalização democrática, poder e tecnologia. 3. ed. Brasília: Observatório do Movimento Pela Tecnologia Social na América Latina/centro de Desenvolvimento Sustentável - CDS, 2010. cap. 1. p. 39-51.

FERNANDES, Josicélia Dumêt; XAVIER, lara de Moraes; CERIBELLI, Maria Isabel Pedreira de Freitas; BIANCO, Maria Helena Cappo; MAEDA, Dirce; RODRIGUES, Michele V. Diretrizes curriculares e estratégias para implantação de uma nova proposta pedagógica. Revista Escola de Enfermagem da USP, São Paulo, v. 39, n. 4, p. 443-449, dez. 2005. Disponível em:

http://www.scielo.br/scielo.php?script=sci_arttext\&pid=S0080-

62342005000400011\&lng=en\&nrm=iso. Acesso em: 04 nov. 2019.

FREITAS, Marcos Costa de. Heranças e desafios históricos do ensino de design gráfico no Brasil. In: CONGRESSO BRASILEIRO DE PESQUISA E DESENVOLVIMENTO EM DESIGN, 9., São Paulo. Anais [...]. São Paulo: [s.n.], 2010.

HATADANI, Paula; ANDRADE, Raquel; SILVA, José. Um estudo de caso sobre o ensino do Design no Brasil: A Escola Superior de Desenho Industrial (ESDI). In: CONGRESSO BRASILEIRO DE PESQUISA E DESENVOLVIMENTO EM DESIGN, 9., São Paulo. Anais [...]. São Paulo: [s.n.], 2010. 
A tecnologia digital nas Diretrizes Curriculares dos Cursos de Design: um estudo da abordagem tecnológica na formação do futuro profissional

Flavia Lumi Matuzawa, Ana Leticia Oliveira do Amaral, Berenice Santos Gonçalves, Roseli Zen Cerny, Marina Bazzo de Espíndola

JANKEVICIUS, José Vitor; HUMEREZ, Dorisdaia Carvalho de. Conceitos Básicos das Diretrizes Curriculares Nacionais (DCNS) dos cursos de Graduação da Área de Saúde. Conselho Federal de Enfermagem. [S.I.], 25 nov. 2015. Disponível em: http://www.cofen.gov.br/wpcontent/uploads/2015/11/Artigo-Conceitos-B\%C3\%A1sicos-das-Diretrizes-Curriculares-NacionaisDcns-dos-cursosd-Gradua\%C3\%A7\%C3\%A3o-da-\%C3\%81rea-de-Sa\%C3\%BAde.pdf. Acesso em: 04 nov. 2019.

JULIA, Dominique. A cultura escolar como objeto histórico. Revista Brasileira de História da Educação. Campinas, n. 1, p. 9-43, jan./jun. 2001. Disponível em: http://repositorio.unifesp.br/handle/11600/39195. Acesso em: 04 nov. 2019.

LOPES, Wendell Evangelista Soares. Andrew Feenberg e a bidimensionalidade da tecnologia. Revista de Filosofia Aurora, Curitiba, v. 27, n. 40, p. 111-142, abr. 2015. Disponível em: https://periodicos.pucpr.br/index.php/aurora/article/view/550. Acesso em: 04 nov. 2019.

MORAES, Dijon de. Design e complexidade. In: MORAES, Dijon de; KRUCKEN, Lia. Cadernos de estudos avançado em Design. Belo Horizonte: ED-UEMG, 2008.

MORAES, Dijon de. Pós-graduação em design no Brasil: cenários e perspectivas. Estudos em Design. Rio de Janeiro, v. 22, n. 3, p. 01-12, 2014. Disponível em:

https://estudosemdesign.emnuvens.com.br/design/article/view/116. Acesso em: 05 nov. 2019.

MOREIRA, Samantha Cidaley de Oliveira; SALES, Rosemary Bom Conselho; BARBOSA, Paula Glória; AGUILAR, Maria Teresa Paulino. Relações entre a educação para o futuro e o ensino do design na atualidade. In: SYSTEMS \& DESIGN, 2016, València. Anais [...]. València: Universitat Politècnica de València, 2016. p. 1-15. Disponível em:

http://ocs.editorial.upv.es/index.php/IFDP/IFDP/paper/view/3362. Acesso em: 05 nov. 2019.

NIEMEYER, Lucy. Design no Brasil: origens e instalação. Rio de Janeiro: 2AB, 2000.

SACRISTÁN, José Gimeno. O que significa o currículo? In: SACRISTÁN, José Gimeno (org.). Saberes e incertezas sobre o currículo. São Paulo: Penso, 2013. cap. 1. p. 16-35.

SILVA, Danilo Emmerson Nascimento. Reflexões e inquietudes sobre design: aspectos da linguagem e da pedagogia do Desenho Industrial. Projética, Londrina, v. 8, n. 1, p. 59-78, jan./jun. 2017.

Disponível em: http://www.uel.br/revistas/uel/index.php/projetica/article/view/25915. Acesso em: 05 nov. 2019.

SILVEIRA, André Luis Marques da; BERTONI, Cristopher Faoro; RIBEIRO, Vinícius Gadis. Premissas para o ensino superior do design. Design e Tecnologia, Porto Alegre, v. 6, n. 12, p. 21-30, dez. 2016. Disponível em: https://www.ufrgs.br/det/index.php/det/article/view/381. Acesso em: 05 nov. 2019. 